\title{
Assessing the Fracture of a Gravity Dam Under Earthquake Load Using a Mesh-Free Method
}

\author{
R. Das CSIRO Mathematical and Information Sciences, Australia \\ P.W. Cleary CSIRO Mathematical and Information Sciences, Australia
}

\begin{abstract}
Failure by fracture constitutes a dominant mode of failure for concrete gravity dams. In this paper, the fracture of a gravity dam is modelled under fluctuating dynamic loads simulating an earthquake. It uses a mesh-free Lagrangian method called Smoothed Particle Hydrodynamic (SPH). The transient stress field and the resulting damage evolution of the dam structure when subjected to sinusoidal ground motion are investigated. The SPH prediction of the crack initiation location and pattern is consistent with that predicted in the literature using Finite Element Method (FEM). The dynamic responses and the fracture patterns predicted are found to be physically sensible. It is found that the frequency and amplitude of the kinetic energy varies as the structure undergoes progressive fracture.
\end{abstract}

\section{Introduction}

Dams are an integral part of industrial and agricultural infrastructure. Dam failure leads to catastrophic consequences including economic loss and loss of human life. One of the most common causes of failure is the initiation and propagation of cracks leading to fracture. The causes of fracture include factors such as seismic load, uneven settling of foundations, thermal stresses due to temperature variations, residual stresses resulting from chemical reactions during setting and hardening of concrete, etc. Pre-existing internal defects or micro-cracks, driven by these internal and external factors, can cause crack growth which might lead to catastrophic failure of the dam.

In seismic areas dams are designed to withstand two levels of earthquakes, design base earthquake (DBE) and maximum creditable earthquake (MCE) (Calayir and Karaton, 2005b). The prediction of failure of dams under earthquake load is critical for designing these structures. Understanding the failure pattern of dams will assist in evaluating performance in the design stage and enable incorporation of design features in the structure to produce improved fracture resistance. This knowledge will also help estimate the consequences of failure and guide the choice of post-failure measures.

In the past, approaches for studying fracture have been heavily dependent on empirical relations based on laboratory scale experimental data (Fujiwara, 1989). For large scale fracture problems, the extrapolation of this data using a scaling law is not always reliable (Shockey et al., 1974). As a result, the empirical models do not often perform well outside the regimes of the original experiments. Numerical fracture modelling can potentially provide effective solutions for predicting large scale dam failures. Furthermore, a well validated numerical model will allow damage initiation and progression to be rapidly and easily assessed for a wide range of problem parameters, such as geometry, loading, initial defect characteristics and distribution. The parameter values can be extended well beyond the scope of experiments. Modelling can also assist in reworking existing designs for enhanced performance and operation. Effective damage modelling techniques in conjunction with efficient numerical methods have strong potential for increasing our understanding of fundamentals of breakage in dams that can be used in the design and operational stages.

The crucial numerical aspects that need to be addressed when simulating dams are the analysis type, computational framework and damage model. When concrete dams are subjected to design base earthquake load, they usually remain in the elastic range (Calayir and Karaton, 2005b). Development of cracks often leads to elastic-brittle fracture under these conditions. Linear dynamic analysis of dams is valid before cracking. However, with the initiation and propagation of cracks, the configuration and stiffness of the 
structure change non-linearly with the progress of cracking. Consequently, a non-linear transient analysis is essential to predict the seismic response.

Common approaches of modelling fracture of dams are the use of mesh-based methods, such as the Finite Element Method (FEM) (Calayir and Karaton, 2005a) and the Boundary element method (BEM) (Batta and Pekau, 1996). These methods need specific techniques (such as ALE formulations involving adaptive meshing) to model large scale discontinuous breakage. In general, the grid based methods require very fine meshes to model the theoretically singular stress field in the neighbourhood of damage locations (e.g. crack tips) (Aliabadi and Rooke, 1991). As the damage propagates, the structure then needs to be re-meshed to take into account the localised change in geometry in order to avoid accuracy and stability issues relating to poor element quality after large deformation. Automated re-meshing can lead to mesh distortion, numerical diffusion and inaccurate results, especially in gradient computations and can be computationally expensive. The FE based models thus have limitations for predicting the modes of failure as well as post-fracture dynamic behaviour including the fragmentation pattern and the interaction of the fragments with the surrounding structures.

In this paper we investigate the fracture of a typical concrete gravity dam, the well-known Koyna dam in India. The failure of Koyna dam has been studied by many researchers (Ayari and Saouma, 1990; Bhattacharjee and Leger, 1993; Ghrib and Tinawi, 1995; Batta and Pekau, 1996; Guanglun et al., 2000; Calayir and Karaton, 2005a; Calayir and Karaton, 2005b). Early numerical modelling of dam failures heavily relied on discrete crack propagation approaches (Ayari and Saouma, 1990; Bhattacharjee and Leger, 1993). Bhattacharjee and Leger developed a smeared crack model and implemented it for FEM analysis of the twodimensional (2D) seismic response of Koyna Dam (Bhattacharjee and Leger, 1993). This model included strain softening, rotation of fracture bands, and strain rate sensitivity of fracture parameters. However, the base of the dam was assumed to be rigid, and this assumption affected accurate prediction of the dynamic behaviour of the dam. This 2D BEM based single crack propagation model was extended to multiple cracks in modelling the response of the Fongman and Koyna dams under earthquake excitation (Batta and Pekau, 1996). The discrete models have the disadvantage that the crack initiation sites need to be explicitly specified in the structure, which is often difficult under complex earthquake loads. Also complex crack behaviours, such as interaction, merging and branching between multiple cracks, are difficult to incorporate.

A continuum volume averaged representation of damage means that the cracks can initiate at an arbitrary location and grow and interact according to the stress field. Ghrib and Tinawi used a continuum damage model combined with a constitutive material model for concrete for modelling Koyna dam (Ghrib and Tinawi, 1995). In the FE modelling, they used a mesh-dependent hardening technique to avoid the sensitivity of energy dissipated on element size. Guanglun et al. used a nonlinear crack band theory in conjunction with FEM to predict the fracture of Koyna dam (Guanglun et al., 2000). This approach required the element size to be close to the characteristic size of the crack band of concrete. So this characteristic size needs to be accurately determined prior to generating the FE mesh, which is often difficult to estimate a priori. Also this model involved continuous re-meshing ahead of the crack front with specific orientation of the cracking element edges parallel to the principal stresses to facilitate the simulation of crack extension. This requires a very fine mesh to allow the propagation of damage/cracks to be accurately predicted and avoid mesh dependent fracture pathways.

Here we use a particle based method called SPH to model brittle fracture of Koyna dam. SPH has been traditionally used for modelling fluid flows (Monaghan, 1992; Cleary et al., 2002; Imaeda and Inutsuka, 2002; Cleary et al., 2007). In recent years, there has been a growing interest in applying SPH to a wide variety of solid mechanics problems (Libersky and Petschek, 1990; Wingate and Fisher, 1993; Gray et al., 2001; Gray and Monaghan, 2004; Liu et al., 2004; Cleary et al., 2006). SPH is a mesh-free numerical method, which is used to obtain solutions to systems of partial differential equations. The problem geometry is discretised into 'particles' that represent specific material volumes. For details of SPH fundamentals, see Monaghan (1992, 2005) and Cleary et al. (2007).

A particle method is preferred over traditional mesh-based techniques (e.g. FEM and BEM) for such fracture prediction problems for several reasons. Firstly, nonlinear FE analysis for a fracturing structure can be computationally expensive and may pose stability problems, as the stiffness matrix needs to be assembled in 
each time step and, for a damaged body, it can also become singular leading to analysis failure. The SPH method used here automatically takes into account the geometric nonlinearities of the problem.

One primary advantage of SPH for fracture is that it does not have any underlying grid structure to represent the geometry and so avoids the inaccuracies and instabilities associated with maintaining the integrity and quality of the mesh during large deformation. The mesh-free nature of SPH makes it ideally suited to modelling fracture processes that involve extreme deformations and discontinuities.

One key requirement of fracture simulation is the ability to model the disintegration of solids to produce fragments. One common approach is to explicitly simulate crack propagation with FEM by detaching elements along the sides of propagating cracks, which is accomplished by allowing separation of the coincident (shared) nodes of the adjacent elements as the fracture surface evolves (Aliabadi and Rooke, 1991). This requires a very fine mesh to allow the propagation of damage/cracks to be accurately predicted and avoid mesh dependent fracture pathways. A continuum volume averaged representation of damage means that the cracks are not constrained to propagate along specific element boundaries even for a coarse mesh allowing natural development of the fracture pattern. SPH has the ability to naturally track new free surfaces as they are generated during fracture.

SPH is a Lagrangian method, in which the equations are solved on the particles which are fully advected with the material flow velocities. SPH is able to follow high speed flows and structural motion without the need to include any explicit free surface tracking and is therefore less diffusive. With the use of a Lagrangian reference frame, each particle continuously represents the same volume of material. This provides a natural ability to track material history and history dependent properties of the material (Cleary et al., 2007). SPH has been successfully applied to simulate different types of solid material forming processes (Cleary et al., 2006) using such history tracking capabilities. In general, fracturing is driven by the stressstrain history experienced by the material. Traditional Eulerian methods have difficulties in tracking the stress-strain history on a node by node basis and in predicting the evolution of damage in the specimen. The history tracking ability of SPH provides a natural framework enabling the prediction of damage initiation and crack propagation. The dynamics of damage evolution is then able to be explicitly included in the analysis.

\section{Damage modelling}

Dynamic fracture is a complex phenomenon that involves the interaction between inherent or generated defects (flaws) and transient stress waves propagating within the material (Barker et al., 1978). The key issues in numerical modelling of damage are:

- Representing inherent initial defects in a material.

- Characterising the level of fracture at a specific location in a material.

- Representing damage growth leading to fracture.

We extend the SPH formulation for elastic solid deformation given in (Gray et al., 2001) by combining it with a continuum damage model to simulate brittle fracture. We use a modified form of the well-established Grady and Kipp damage model (Grady and Kipp, 1980). This model is widely used for the prediction of rock damage based on the local stress history and flaw distribution. To characterise the extent of fracture in a volume of material, a scalar damage parameter $D$ is used to indicate the volume averaged micro-fracture of the material represented by each SPH particle. The damage parameter inhibits the transmission of tensile stress between particles and it evolves based on the strain history of each particle. The damage parameter lies between zero and unity. Material with $D=0$ is undamaged/uncracked and is able to transmit the full tensile load, whereas material with $D=1$ is fully damaged and cannot transmit any tensile load, thus creating a partial crack. Contiguous cracked material across a body leads to fragmentation.

Assuming a constant crack growth speed $C_{g}$ the damage $D(t)$ evolution is given by the integral equation:

$$
D(t)=\frac{4}{3} \pi C_{g}^{3} \int_{0}^{t} n^{\prime}(\varepsilon) \&(1-D)(t-\tau)^{3} d \tau
$$

where $\varepsilon$ is the tensile strain, $n^{\prime}$ is the change in number of flaws, $t$ is the current time, $\tau$ is the past times for history integration. 
An approximate differential form of Equation 1 (from Grady and Kipp, 1980) is:

$$
\frac{d D^{1 / 3}}{d t}=\frac{(m+3)}{3} \alpha^{1 / 3} \varepsilon^{m / 3}
$$

where $\alpha$ is a material constant calculated from three material fracture parameters $k, m$, and $C_{g}$ :

$$
\alpha=\frac{8 \pi C_{g}^{3} k}{(m+1)(m+2)(m+3)}
$$

The original Grady-Kipp model is valid only for one-dimensional plain strain problems. For use in two or three-dimensions, the full stress-strain field needs to be reduced to the one-dimensional analysis framework outlined in (Grady and Kipp, 1980). One proposed method replaces the longitudinal strain by the volumetric strain (average of the three longitudinal strain components) (Thorne et al., 1990). This poses difficulties when some of the longitudinal strain components are compressive and some are tensile, and their magnitudes are such that the resulting volume strain is compressive. In this case, no damage evolution will occur under a compressive strain according to this damage model, but in reality damage accumulation should take place due to the tensile component(s) of the strain tensor. To avoid this problem, Melosh et al. introduced an effective tensile strain given by (Melosh et al., 1992):

$$
\varepsilon_{\text {eff }}=\sigma_{\max } /\left(K+\frac{4}{3} G\right)
$$

where $\sigma_{\max }$ is the maximum positive principal stress, and $K$ and $G$ are the bulk modulus and the shear modulus of the material.

Damage then accumulates when the effective strain exceeds a threshold value $\varepsilon_{\min }$ :

$$
\varepsilon_{\min }=(V k)^{-1 / m}
$$

where $V$ is the volume of each SPH particle.

This effective strain is used in the evolution Equation (2) to predict the damage state of the material. So for damage evolution in SPH we use the combination of the differential version of the Grady-Kipp model (Equation (2)) and the effective tensile strain (Equation (4)). The total stress tensor is rotated into its principal co-ordinate frame where the tensile stress is easiest to identify and then to scale only the tensile components of the principal stresses. These are rotated back to the world co-ordinate frame to give the postdamage stresses used in the stress evolution and momentum equations in (Gray et al., 2001).

\section{Dynamic fracture simulation}

\subsection{Modelling approach and problem configuration}

Here we investigate the response of Koyna dam under periodic vibration of the base to study the fracture initiation and propagation pattern using 2D SPH simulations. This simplified example can provide insight into the key mechanisms of dam fracture under earthquake for similar categories of gravity dams.

Dam-foundation interaction is a crucial aspect in the dynamic behaviour of dams. Many approaches consider the foundation (base) to be infinitely rigid (Chopra and Gupta, 1982). However, the elasticity of the base can strongly affect the dynamic response and hence the fracture pattern. So here the dam base is modelled as a flexible (elastic) material and the dam is treated as an elastic-brittle solid. The bottom part of the dam base is rigidly anchored with the ground.

Figure 1 shows a 2D vertical cross-section of the dam modelled in this study. The geometries of the dam and the base are taken from (Calayir and Karaton, 2005b). The dam is $103 \mathrm{~m}$ high and $70 \mathrm{~m}$ wide at the base and is made of concrete with bulk modulus of $16.7 \mathrm{GPa}$, shear modulus of $8.1 \mathrm{Gpa}$, and density of $2400 \mathrm{~kg} / \mathrm{m}^{3}$. The Weibull damage parameters for concrete used in the Grady-Kipp model are $k=5.27 \times 10^{18}$ and $m=9.5$ (Melosh et al., 1992). The base of the dam usually consists of a harder and stiffer material (rock). It is assumed to be damage resistant and undergoes elastic deformation only. Here the base material is basalt rock 
with bulk modulus of $58.3 \mathrm{Gpa}$, shear modulus of $26.9 \mathrm{Gpa}$, and density of $2900 \mathrm{~kg} / \mathrm{m}^{3}$. The dam and the base are discretised with particles of one metre spacing, giving total 5856 particles in the 2D simulation. Based on these material properties, the sound speeds are 2638 and $4929 \mathrm{~m} / \mathrm{s}$ in the dam and the base respectively.

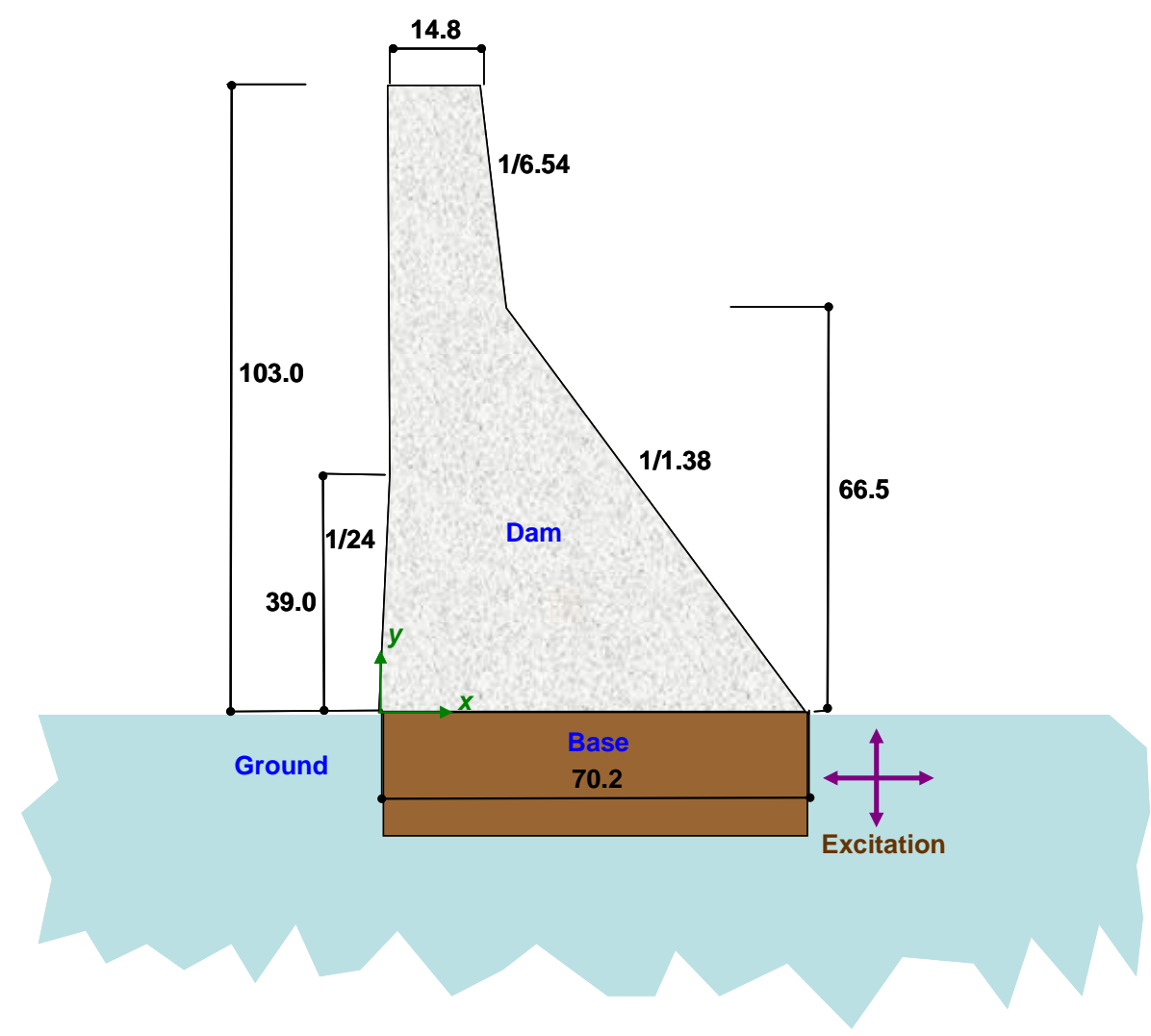

Figure 1 Geometry of the 2D section of Koyna dam (all dimensions are in metres and taken from Calayir and Karaton, 2005b)

The base of the dam is subjected to fluctuating loads in the horizontal and vertical directions to simulate the ground motion during an earthquake. In this preliminary study, the aim is to consider the development of damage, fracture pattern, and the transfer and variation of energy during fracture. To facilitate easy evaluation and analysis of these structural responses, a simple dynamic load is used to simulate the earthquake, rather than using more realistic random earthquake accelerograms. The base of the dam is subjected sinusoidal loading in the horizontal and vertical directions. The amplitude, frequency and phase in both the directions are the same resulting in a sinusoidal ground excitation at an angle of $45^{\circ}$. The fracture pattern obtained (Calayir and Karaton, 2005b) using the real Koyna earthquake record is moderate and consists of localised cracks. In this study, the amplitude of the ground vibration is chosen to be six times higher than the average amplitude encountered in the 1967 Koyna earthquake (Calayir and Karaton, 2005b) to demonstrate that SPH can potentially simulate extreme deformation and fragmentation, beyond what occurred in the case of Koyna earthquake. The amplitude and frequency of the acceleration of the base are chosen to be $1.12 \mathrm{~g}$ and $10 \mathrm{~Hz}$ respectively.

\subsection{Crack propagation and dynamic fracture}

Figure 2 shows the early stress wave propagation in the dam as it oscillates under the base motion. The particles are coloured so that the blue-red colour range corresponds to a von Mises stress range of 0-70 MPa. The interaction of the ground with the base during oscillating motion alternately compresses the base of the dam on either side. This leads to the generation of stress waves at the base that propagate towards the upper part of the structure (Figure 2(a)-(b)). The elastic waves reflect from the free surfaces of the dam (Figure 2(c)). The reflected waves propagate back inwards and interfere with the (newly generated) incident 
waves from the base. This creates an interacting wave pattern by superposition of the incident and reflected waves. This early stress wave loading leads to high stresses in the dam.

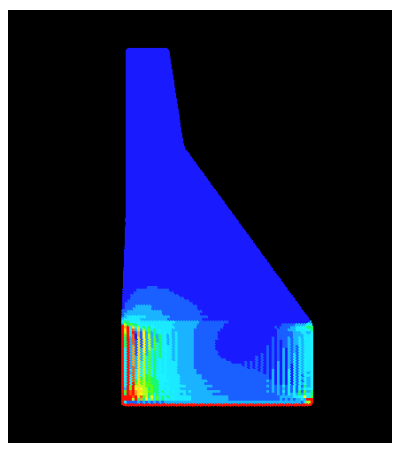

(a) $t=20 \mathrm{~ms}$

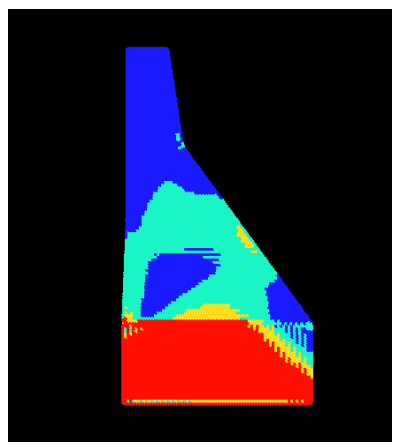

(c) $t=60 \mathrm{~ms}$

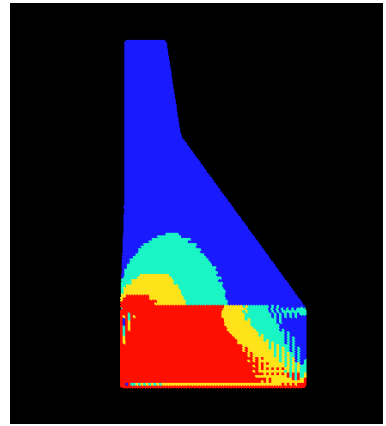

(b) $t=40 \mathrm{~ms}$

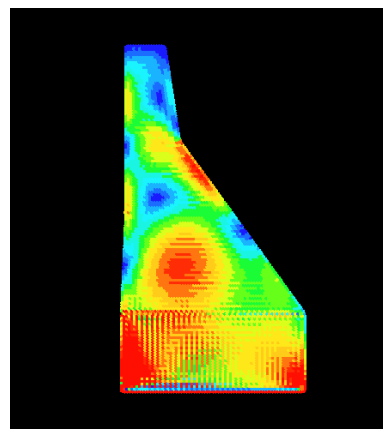

(d) $t=80 \mathrm{~ms}$

VM stress 0.0

$7.00 \mathrm{E}+07$

Figure 2 Stress wave propagation in the early stages due to ground excitation (coloured by von Mises stress (Pa))

Figure 3 shows the evolution of the von Mises stress (left column) and damage (right column) in the dam as it undergoes fracture. The particles are coloured so that the blue-red colour range corresponds to a von Mises stress range of $0-12.5 \mathrm{GPa}$ and damage range of zero to one.

Due to stress wave loading, high stress concentrations are generated at the point on the right side of the dam at which the downstream (right side) slope of the wall changes discontinuously (Figure 3(a) left). This location acts as the initial damage initiation site, and the first crack is created here at around $0.1 \mathrm{~s}$. This crack propagates from right to left to the middle of the section and then turns upwards and propagates towards the left face (Figure 3(a) right). At $0.15 \mathrm{~s}$, another crack originates from the pathway of the first crack, propagates downwards and reaches the left-hand surface of the dam. These cracks will be termed as the 'upper cracks'. The initial crack initiation site (Figure 3(a)) predicted by SPH matches with those obtained in the literature using realistic earthquake acceleration records (Bhattacharjee and Leger, 1993; Calayir and Karaton, 2005a).

The propagation of upper cracks is accompanied by the initiation of a crack (called the 'middle crack') from the inclined surface of the right face due to the high stress generated at the right surface. This crack starts at an orientation normal to the surface, propagates towards the interior, and then branches out into two cracks at $0.20 \mathrm{~s}$ (Figure 3(b-c)) that travel leftwards and downwards respectively. The left one moves slightly upwards and reaches the left-hand surface, where it meets the tip of one of the upper cracks. At $0.26 \mathrm{~s}$ another crack emanates from the pathway of this middle crack and reaches the left face at $0.30 \mathrm{~s}$ (Figure 3(c)). The bottom crack branch propagates a short distance downwards and then also branches into two cracks. The left branch bends upwards and the right branch propagates down (Figure 3(d)). Immediately before reaching the right inclined face, two more branches are created from this (right branch) crack. One branch intersects the right face perpendicularly, and the other one propagates further down and bends sideways, just before the base, to meet the right face perpendicularly (Figure 3(e)). 

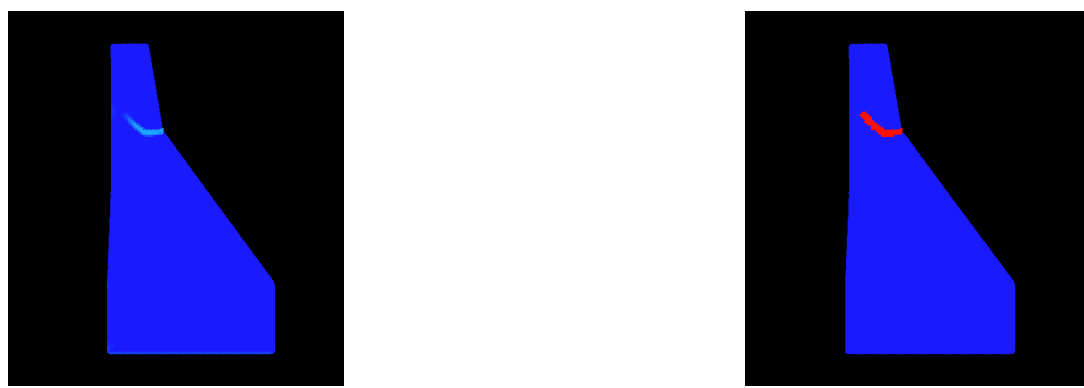

(a) $t=0.12 \mathrm{~s}$
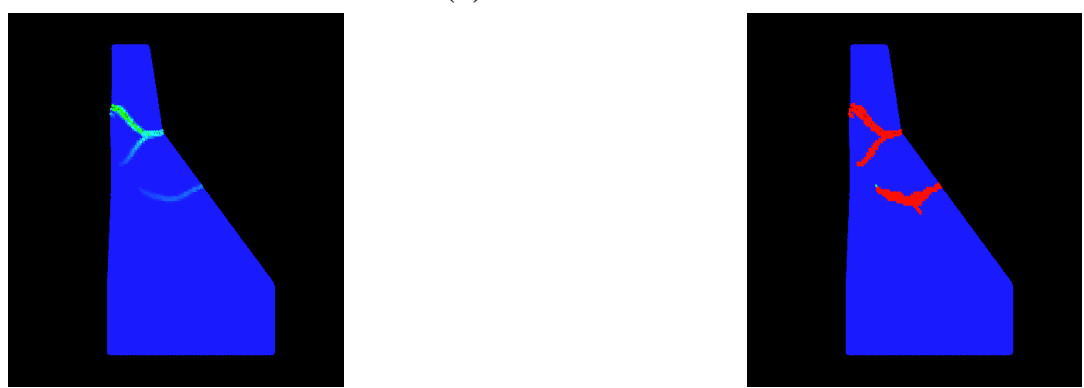

(b) $t=0.20 \mathrm{~s}$
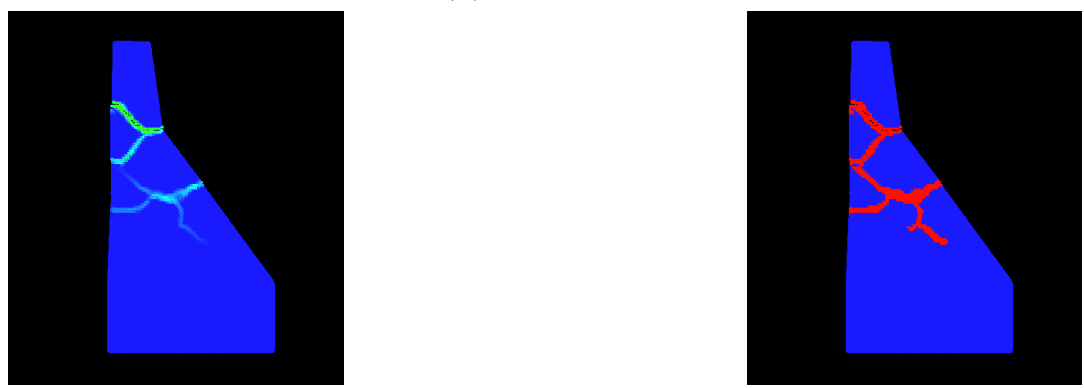

(c) $t=0.30 \mathrm{~s}$
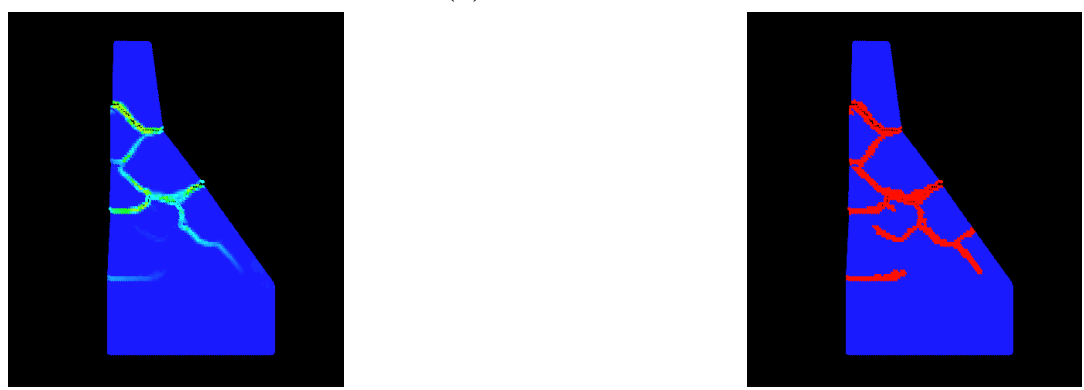

(d) $t=0.40 \mathrm{~s}$
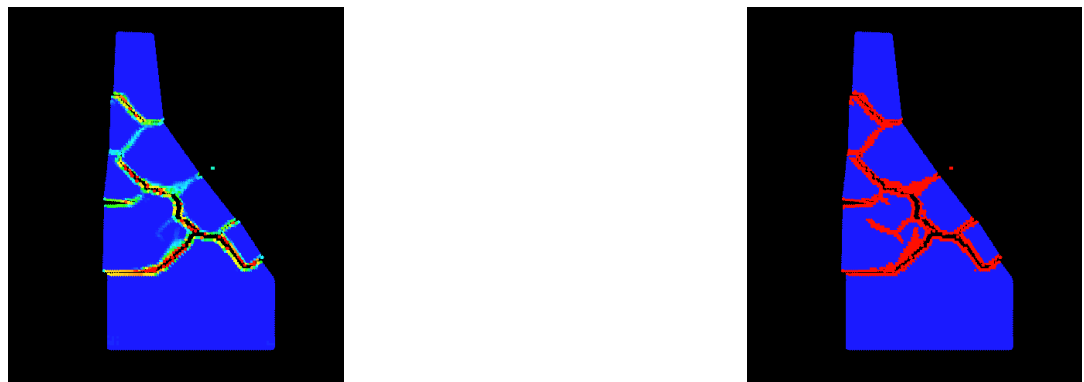

(e) $t=1.0 \mathrm{~s}$

VM stress 0.0

$1.25 \mathrm{E}+10$

Damage $\quad 0.0$

Figure 3 Fracture pattern of the dam subjected to base motion in the horizontal and vertical directions (left: coloured by von Mises stress (Pa) and right: coloured by damage) 
Due to lateral vibration, high stresses are developed near the intersection of the base and the dam. As a result, a 'lower crack' originates from the left intersection point at $0.38 \mathrm{~s}$ propagates horizontally up to onethird of the width of the base and then bends to propagate upwards intersecting the existing network of cracks. This is the longest crack in the structure that partially separates the dam from its base (Figure 3(e)).

The von Mises stress is very high along the cracks (fracture planes), whereas the stress is very low within each region bounded by networks of cracks, since after fracture no tensile stress can be transmitted across the crack planes into these regions.

From the fracture pattern observed above, we can identify the following characteristics of the dynamic crack propagation in the dam:

- The cracks originate normal to the wall surface because the normal stress is very small close to the free surface and the tangential stress is approximately equal to both the maximum principal stress and the von Mises stress. This makes the paths normal to the surfaces most critical along which the cracks preferably initiate and grow normal to the direction of the maximum principal stress.

- As the cracks propagate towards the interior, they sometimes branch out one or more times to produce pairs of cracks in each case of branching. These new cracks (branches) propagate towards the dam surfaces because this provides the path of least resistance to fracture (along which fracture will consume the minimum energy) (Anderson, 1991).

- During the periodic dynamic loads, the interaction of the impinging stress waves with the rarefaction compression waves reflected from the free surfaces slows the normal propagation of cracks. So while approaching the free surfaces, this decelerating effect may cause bending of the advancing crack fronts (depending on the local stress wave loading), as observed for the uppermost crack on the left face and the lowermost crack on the right face (Figure 3(e)). This phenomenon is known as 'crack arrest' and has been observed previously in collision fracture problems (Das and Cleary, 2008a; Das and Cleary, 2008b). Many structural designs in fact incorporate features that facilitate resistance to fracture by decelerating the propagation of cracks.

\subsection{Variation of damage and energy of the dam}

Figure 4 shows the variation of the fraction of damage in the dam. The damage accumulation commences at $0.09 \mathrm{~s}$, and the increase in damage occurs in two distinct stages, labelled as $\mathrm{AB}$ and $\mathrm{BC}$ in Figure 4. In the first stage, the damage increases at a moderate rate until $0.37 \mathrm{~s}$ where it reaches a value of 0.08 (point $\mathrm{B}$ ). The damage then increases sharply reaching 0.12 at $0.44 \mathrm{~s}$. It then reaches a plateau corresponding to the end of fracturing of the dam structure. The initial rise in damage (from A to B) represents its accumulation in the upper and central region of the dam during the initiation and subsequent growth of the cracks. The rate of damage rise varies with time depending on the current fractured state of the structure and the crack propagation pattern at any given time. Damage alternately increases and remains uniform for a short period during this first stage. The increase in damage occurs when the existing cracks grow and branch into multiple cracks, or when new cracks initiate either from the surfaces of the dam or from the pathways of the existing cracks. The cessation of damage growth for a short period after each rise represents the time during which the existing crack growth terminates. In the second stage from B to C, the longest (lower) crack initiates from the left side of the dam-base junction, propagates first along the interface, and then towards the middle causing rapid fracture. This leads to the rapid increase in damage from 0.08 to 0.12 up to $0.44 \mathrm{~s}$. At point $\mathrm{C}$, the fracturing effectively ceases and the damage remains unchanged thereafter. 


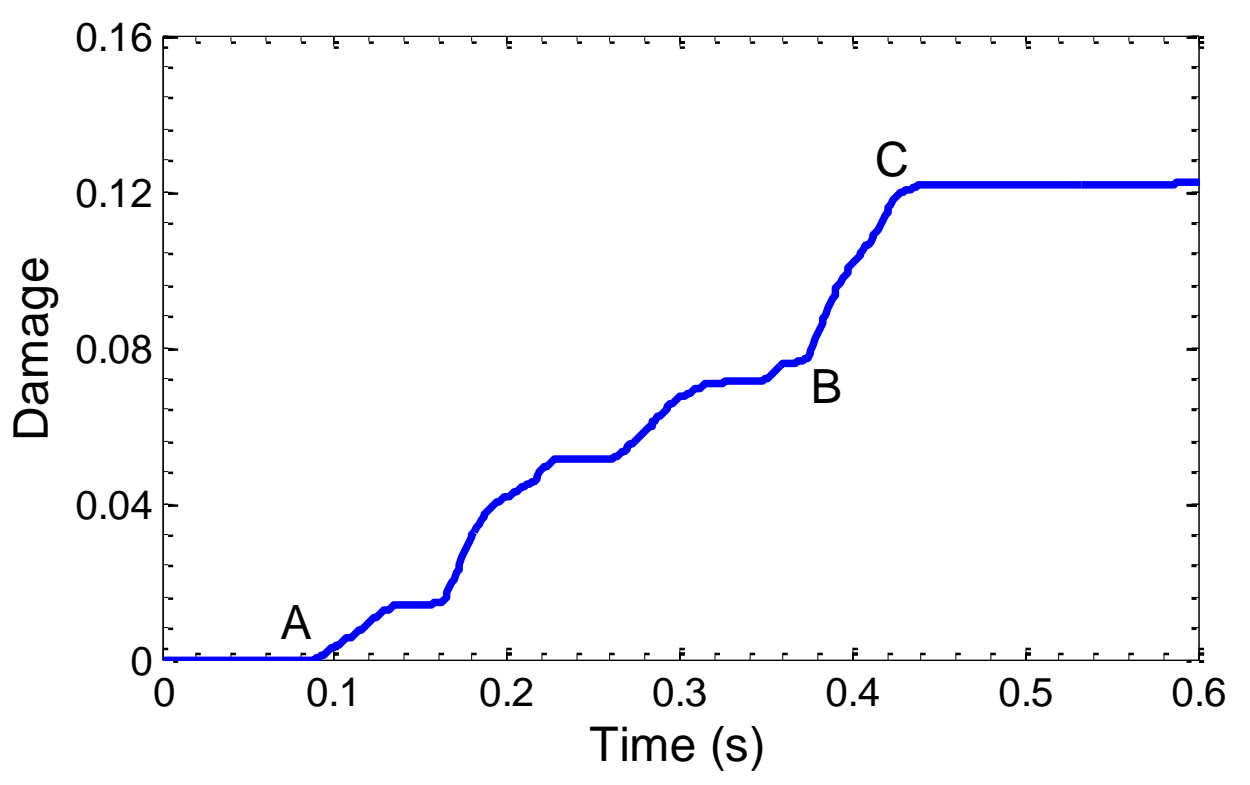

Figure 4 Variation of the average damage in the dam with time

One key factor that affects fracture under earthquake load is the energy transfer to the structure from the ground. The energy transferred to the dam is partitioned in three ways: (a) the energy dissipated in fracture, (b) the kinetic energy of the accelerated fragments and the dam, and (c) the elastic energy of the dam.

Figure 5a shows the variation of kinetic energy of the dam-base structure, which fluctuates throughout the fracturing process under the sinusoidal ground excitation. Kinetic energy is continuously transferred to the structure from the oscillating ground. As the dam progressively undergoes fracture, the configuration and stiffness of the resulting structure are altered over time. This results in non-uniform oscillations in the kinetic energy. The kinetic energy initially increases monotonically up to $0.09 \mathrm{~s}$ prior to the commencement of fracture. It then starts fluctuating and the moving averaged value of the kinetic energy over each cycle (termed as the 'mean kinetic energy') increases until $0.35 \mathrm{~s}$, at which it reaches its maximum value of $226 \mathrm{MJ}$, marked as point $\mathrm{P}$. This corresponds to the first stage of damage indicated by label AB in Figure 4 . In this stage, the kinetic energy imparted to the dam from the base exceeds the energy consumed in fracture, so the mean kinetic energy increases up to the end of the first stage of damage. The second stage of rapid damage is accompanied by the propagation of the lower (base) crack, and leads to higher energy being consumed in fracturing. Also, due to fracture, some parts of the dam are separated as fragments from the main structure (which are connected to the base). The loads on these fragmented regions are relatively low because tensile forces cannot be transmitted across the fracture planes. As a result, kinetic energy can no longer be transferred to these fragments from the base. Consequently, the mean kinetic energy decreases up to one s (Point Q). It subsequently increases somewhat reaching a value of $136 \mathrm{MJ}$ at point $\mathrm{R}$ (at $1.6 \mathrm{~s}$ ) because of the collisions between fragments and the main dam structure. The mean energy subsequently shows a decreasing trend as the colliding fragments separate from the main structure.

Figure 5(b) shows the FFT of the kinetic energy which has a number of major and minor peaks. The highest peak (point $\mathrm{K}_{1}$ ) at frequency $0.5 \mathrm{~Hz}$ corresponds to the frequency of the mean kinetic energy variation, as seen in Figure 5(a). The frequencies at peaks $\mathrm{K}_{2}-\mathrm{K}_{5}$ are 10.0, 19.8, 30.1 and 40.2 Hz. These are very close to the forcing (excitation) frequency of $10 \mathrm{~Hz}$ and its harmonics. The power spectral density (PSD) associated with the second harmonic (point $\mathrm{K}_{3}$ ) is comparatively lower. As a result of progressive crack propagation, the kinetic energy of the structure oscillates with varying frequencies and amplitudes depending on the damage level at any given time. When the natural frequency of a given structural configuration during the fracture process becomes close to the forcing (ground) frequency or its harmonics, a relatively high peak is obtained, as observed at points $\mathrm{K}_{2}, \mathrm{~K}_{4}$, and $\mathrm{K}_{5}$. The other frequencies with relatively minor peaks represent the natural frequencies of the structure for different fractured states. 


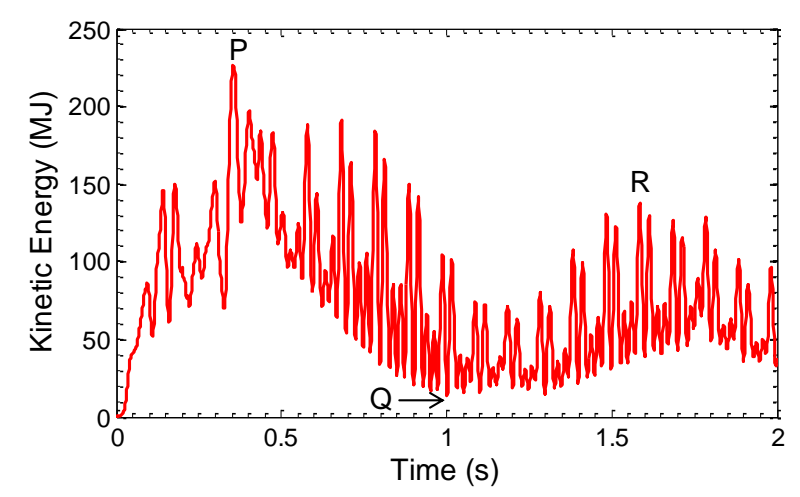

(a)

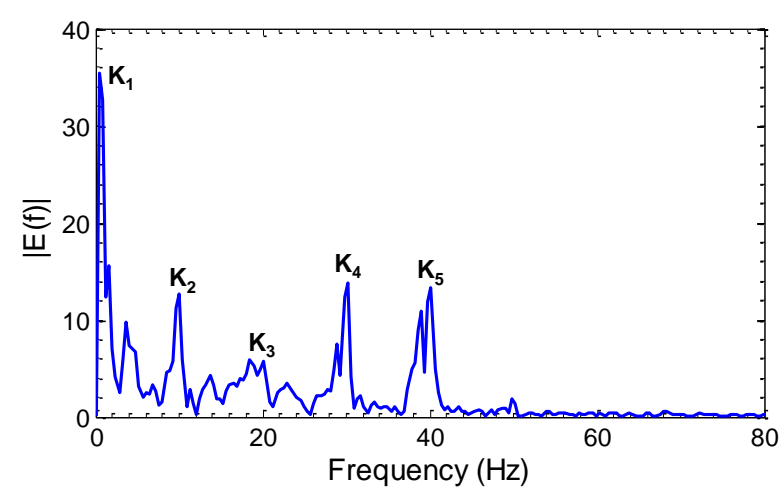

(b)

Figure 5 (a) Variation of the kinetic energy with time; (b) FFT of the kinetic energy showing the frequency distribution (the excitation frequency is $10 \mathrm{~Hz}$ )

Figure 6 shows the velocity distribution of the dam structure and fragments (with dark blue being stationary and red being $10 \mathrm{~m} / \mathrm{s}$ ). The velocity of the fragments is strongly dependent on the fractured state of the dam. Figure 6(a) shows the velocity distribution at $0.35 \mathrm{~s}$ when the kinetic energy reaches its maximum value (labelled as point $\mathrm{P}$ in Figure 5(a)). The lower portion of the dam, attached to the base, has the highest velocity. The fragmented middle and the upper sections have comparatively lower velocities. With continued fracture, more regions of the dam separate from the main structure. These fragments lose contact with the lower part of the dam, which prevents the transfer of kinetic energy from the main structure and leads to reduction of their velocities. Figure 6(b) shows the velocity distribution when the (mean) kinetic energy is at minimum (point Q in Figure 5(a)). Afterwards the fragments travel vertically under gravity and collide with those below and the main dam structure (connected to the base). This increases the velocity (hence the kinetic energy) of the fragments (Figure 6(c)), which corresponds to point R in Figure 5(a). This is followed by post-collision separation of the fragments leading to a decline in the kinetic energy of the structure (Figure 6(d)).

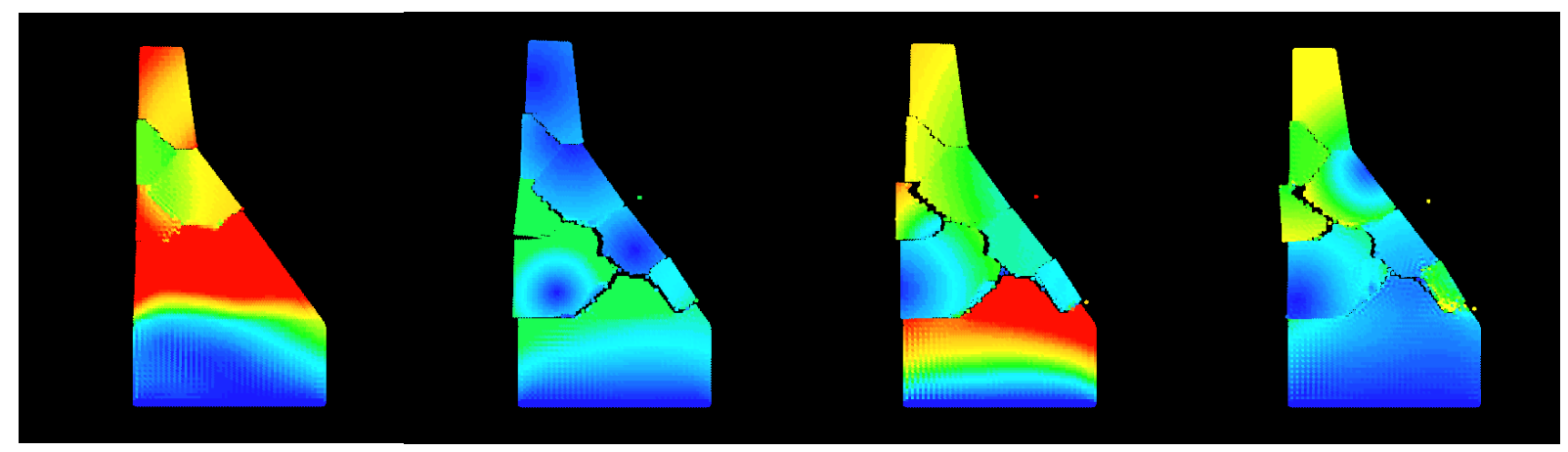
(a) $t=0.35 \mathrm{~s}$
(b) $t=1.0 \mathrm{~s}$
(c) $t=1.6 \mathrm{~s}$
(d) $t=2.0 \mathrm{~s}$

$\begin{array}{lll}\mathrm{V} & 0.0 & 10.00\end{array}$

Figure 6 Velocity distribution of the dam and fragments with progressive fracture 


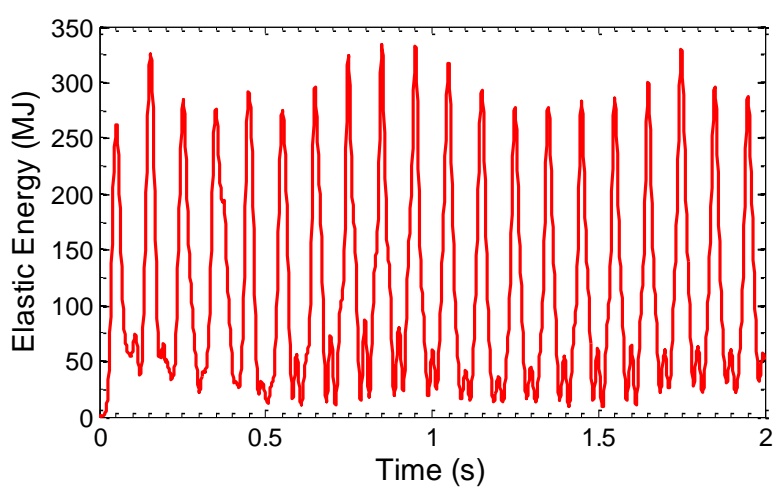

(a)

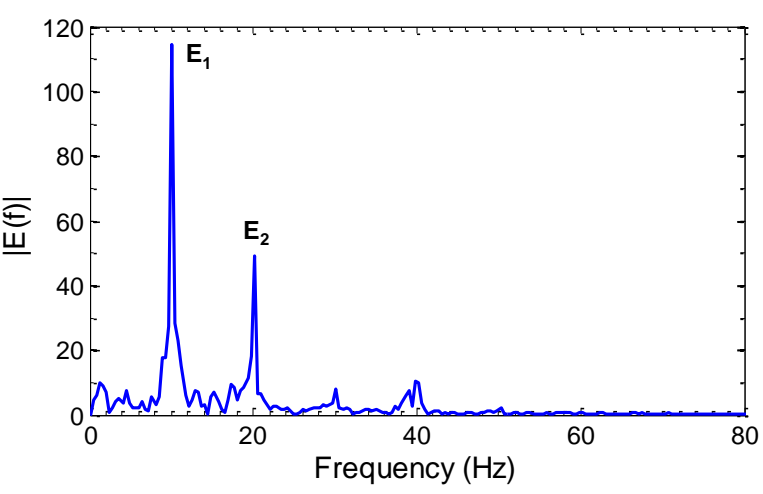

(b)

Figure 7 (a) Variation of the elastic energy with time; (b) FFT of the elastic energy showing the frequency distribution (the excitation frequency is $10 \mathrm{~Hz}$ )

Figure 7(a) shows the variation of the elastic energy of the dam and the base. With the oscillation of the ground, the base of the dam is elastically compressed alternately on either side and from the bottom, leading to oscillations in the elastic energy. The oscillations remain fairly uniform, which shows that only a small fraction of the elastic energy is dissipated in fracturing. The variation in the amplitude and frequency over time is considerably less than that of the kinetic energy (Figure 5). The amplitude of the elastic energy varies between 313 and $253 \mathrm{MJ}$ throughout the fracture process. Since the base is damage resistant, it only deforms elastically and does not consume any energy in fracturing. The energy imparted to the base is partly transmitted to the dam structure above and partly stored as elastic compression energy. When the dam undergoes brittle fracture it stores very little elastic energy. The majority of the total elastic energy is stored in the base with only small dissipation of the elastic energy during fracture. Figure 7(b) shows the oscillation frequencies of the elastic energy. The major peak $E_{1}$ and the minor peak $E_{2}$ correspond to the frequencies of 10 and $20 \mathrm{~Hz}$ respectively. The major frequency matches with the forcing frequency $(10 \mathrm{~Hz})$ of the ground excitation, and the minor frequency represents the second harmonic. This confirms that the fracturing of the upper dam structure does not considerably affect the elastic energy variation, and the energy expended during damage is primarily contributed by the kinetic energy of the dam.

\section{Conclusions}

Large scale fracture of a concrete gravity dam under earthquake-like ground excitation has been modelled using SPH. The initial crack initiation location agrees well with that predicted by FEM in the literature. The grid-less Lagrangian framework of SPH has enabled prediction of phenomena beyond that which is possible with traditional mesh-based approaches, such as large scale extreme deformation and discontinuous fracture.

The fracture pattern of the dam predicted by SPH is physically sensible. The initiation and growth of the cracks separate different parts of the dam from the base. This starts with the complete detachment of the crest block (uppermost part) of the dam in the early stage. This is followed by fracture of the middle section as multiple cracks propagate through-the-width of the dam. Finally, the lower base crack grows along the dambase interface leading to the catastrophic failure of the structure.

The dynamic crack propagation has the following characteristics:

- The cracks initiate from the surfaces of the dam normal to the direction of the maximum principal stress, which is approximately tangential to the surfaces.

- Near the surfaces, crack growth is slowed by reflecting compressive stress waves. This sometimes causes the cracks to bend sideways, and to branch into multiple cracks.

- During the propagation into the dam structure, the cracks branch out to generate a pair of cracks, eventually creating multiple cracks. Most of these cracks propagate through the structure up to the dam wall surfaces and produce fragments. 
- The von Mises stress distribution obtained in the dam is consistent with the fracture pattern observed.

- The damage in the structure varies depending on the crack propagation pattern and the resulting network of cracks throughout the structure.

- During the ground excitation, the energy transferred to the base is primarily stored as elastic compression energy, and some is then transferred to the upper dam structure. The total energy transferred to the upper dam structure is partly consumed in fracturing the dam and the rest is converted to kinetic energy of the un-fractured part of the structure and the fragments, with a small amount is stored as elastic energy. The distribution of the energy influences the fracture pattern and the size of the fragments.

Understanding the key mechanisms of fracture and the resulting fragmentation process in gravity dams provides a basis for improving the design features to generate more fracture resistant structure. It appears that SPH may be an effective tool for use in the initial design stage. A number of designs can be rapidly assessed under a variety of load spectrums to identify the optimal configuration of the structure. Moreover, during the operation of a dam, the response of the structure and the in-service loads can be monitored and can be feedback to the design inputs. This can then be used for more accurate prediction of fracture critical locations, failure pattern and post-failure dynamic response of the structure. This will enable us to take appropriate measures, including re-work of an existing design and incorporation of additional reinforcements, to resist failure, to reduce the extent of failure or to alter the failure pattern to minimise economic and social impacts.

\section{References}

Aliabadi, M.H. and Rooke, D.P. (1991) Numerical Fracture Mechanics, Computational Mechanics Publications and Kluwer Academic Publishers.

Anderson, T.L. (1991) Fracture Mechanics: Fundamentals and Applications, CRC Press.

Ayari, M.L. and Saouma, V.E. (1990) Fracture mechanics based seismic analysis of concrete gravity dams using discrete cracks, Engineering Fracture Mechanics, 35, Publisher, pp. 587-598.

Barker, D.B., Fourney, W.L. and Dally, J.W. (1978) The influence of stress waves on explosive induced fragmentationborehole crack network, 19th U.S. Symp. on Rock Mech., Lake Tahoe.

Batta, V. and Pekau, O.A. (1996) Application of boundary element analysis for multiple seismic cracking in concrete gravity dams, Earthquake Engineering and Structural Dynamics, 25, Publisher, pp. 15-30.

Bhattacharjee, S.S. and Leger, P. (1993) Seismic cracking and energy dissipation in concrete gravity dams, Earthquake Engineering and Structural Dynamics, 22, Publisher, pp. 991-1007.

Calayir, Y. and Karaton, M. (2005a) A continuum damage concrete model for earthquake analysis of concrete gravity dam-reservoir systems, Soil Dynamics and Earthquake Engineering, 25, Publisher, pp. 857-869.

Calayir, Y. and Karaton, M. (2005b) Seismic fracture analysis of concrete gravity dams including dam-reservoir interaction, Computers and Structures, 83, pp. 1595-1606.

Chopra, A.K. and Gupta, S. (1982) Hydrodynamic and foundation interaction effects in frequency response functions for concrete gravity dams, Earthquake Eng. Struct. Dynam., 10, Publisher, pp. 89-106.

Cleary, P., Ha, J., Alguine, V. and Nguyen, T. (2002) Flow modelling in casting processes, Applied Mathematical Modelling, 26, Publisher, pp. 171-190.

Cleary, P.W., Prakash, M. and Ha, J. (2006) Novel applications of smoothed particle hydrodynamics (SPH) in metal forming, Journal of Materials Processing Technology, 177, Publisher, pp. 41-48.

Cleary, P.W., Prakash, M., Ha, J., Stokes, N. and Scott, C. (2007) Smooth particle hydrodynamics: status and future potential, Progress in Computational Fluid Dynamics, 7, Publisher, pp. 70-90.

Das, R. and Cleary, P.W. (2008a) Modelling 3D fracture and fragmentation in a thin plate under high velocity projectile impact using SPH, 3rd SPHERIC workshop, Lausanne, Switzerland.

Das, R. and Cleary, P.W. (2008b) Modelling brittle fracture and fragmentation of a column during projectile impact using a mesh-free method, 6th International Conference on CFD in Oil and Gas, Metallurgical and Process Industries Trondheim, Norway.

Fujiwara, A. (1989) Experiments and scaling laws for catastrophic collisions, Publisher, pp. 240-265.

Ghrib, F. and Tinawi, R. (1995) Application of damage mechanics for seismic analysis of concrete gravity dams, Earthquake Engineering and Structural Dynamics, 24, Publisher, pp. 157-173.

Grady, D.E. and Kipp, M.E. (1980) Continuum modelling of explosive fracture in oil shale, International Journal of Rock Mechanics and Mining Sciences and Geomechanics Abstracts, 17, Publisher, pp. 147-157. 
Gray, J.P. and Monaghan, J.J. (2004) Numerical modelling of stress fields and fracture around magma chambers, Journal of Volcanology and Geothermal Research, 135, Publisher, pp. 259-283.

Gray, J.P., Monaghan, J.J. and Swift, R.P. (2001) SPH elastic dynamics, Computer Methods in Applied Mechanics and Engineering, 190, Publisher, pp. 6641-6662.

Guanglun, W., Pekau, O.A., Chuhan, Z. and Shaomin, W. (2000) Seismic fracture analysis of concrete gravity dams based on nonlinear fracture mechanics Engineering Fracture Mechanics, 65, Publisher, pp. 67-87.

Imaeda, Y. and Inutsuka, S-I. (2002) Shear Flows in Smoothed Particle Hydrodynamics, The Astrophysical Journal, 569, Publisher, pp. 501-518.

Libersky, L.D. and Petschek, A.G. (1990) Smooth particle hydrodynamics with strength of materials, In Advances in the Free-Lagrange Method (eds) Trease and Crowley, Berlin: Springer.

Liu, Z.S., Swaddiwudhipong, S. and Koh, C.G. (2004) High velocity impact dynamic response of structures using SPH method, International Journal of Computational Engineering Science, 5, Publisher, pp. 315-326.

Melosh, H.J., Ryan, E.V. and Asphaug, E. (1992) Dynamic fragmentation in impacts: hydrocode simulation of laboratory impacts, Journal of Geophysical Research, 97, Publisher, pp. 14735-14759.

Monaghan, J.J. (1992) Smoothed particle hydrodynamics, Ann. Rev. Astron. Astrophys., 30, Publisher, pp. 543-574.

Monaghan, J.J. (2005) Smoothed particle hydrodynamics, Rep. Prog. Phys., 68, Publisher, pp. 1703-1759.

Shockey, D.A., Curran, D.R., Seaman, L., Rosenberg, J.T. and Petersen, C.F. (1974) Fragmentation of Rock under Dynamic Loads, International Journal of Rock Mechanics and Mining Sciences, 11, Publisher, pp. 303-317.

Thorne, B.J., Hommert, P.J. and Brown, B. (1990) Experimental and computational investigation of the fundamental mechanisms of catering, 3rd International Symposium on Rock Fragmentation by Blasting, Brisbane, Australia, pp. 117-124.

Wingate, C.A. and Fisher, H.N. (1993) Strength Modeling in SPHC, Los Alamos National Laboratory, Report No. LA-UR-93-3942. 
